\title{
Discriminant Analysis on Complex Material and Variable Structure System Life
}

\author{
Jianhua $\mathrm{Du}^{1, \mathrm{a}}$, Shimeng $\mathrm{Xu}^{2}$, Runbo $\mathrm{Ma}^{2}$, Lei Gong ${ }^{2}$ and Haoxu Wang ${ }^{1, \mathrm{~b}}$ \\ ${ }^{1}$ Department of Scientific Research, Academy of Armored Force Engineering, Beijing 100072, China \\ ${ }^{2}$ Department of Fundamental Courses, Academy of Armored Force Engineering, Beijing \\ 100072,China \\ adjh619@sina.com, bwanghaoxu@nudt.edu.cn
}

\begin{abstract}
Keywords: Complex material system life; Variable structure; Categorizing data; Discriminant analysis; Criterion rules; Simulation method.

Abstract. In this paper, the discriminant categorization on complex material and variable structure system life are given, and there are two kinds of variable structure on complex material system life based on the empirical data and the simulating data and the evaluating and analyses rooted in actual system. Here, according to the two kinds of variable structure with the corresponding data, complex material system lives are categorized based on principal component analysis and classification discriminant analysis, and the front is used to predigest data, the latter is used to categorize data. Thus, the system life data might be categorized as the two kinds of variable structure with runtime and environment based on system life indexes, the precise radio is, and the misjudged radio and the undetermined ratio are summed as $20 \%$.
\end{abstract}

\section{Introduction}

In resent two decades, the studies on complex material system life have achieved quiet great progresses, and the main excellences of these progresses are in a large scale intellectualized calculation and analysis under life data of complex system ${ }^{[1-4]}$. Here gives some examples, such as, network analysis and computation, optimal design of system life, graphic method and data resources visualization, various of intellectual and optimal algorithms, instantaneous tracking technologies, and so on. However, there are still many of troublesome and rudimental issues of complex system life, and that are at least not put across up to the present. Among the issues, it is most prominent essential issue that the initialized data of complex material system life are felicitously disposed, so that subsequent design and analysis on complex material system life might be efficaciously realized. Contrarily, if there are some of defaults and shortages in the felicitous dispose of initialized data on complex material system life, the farther analysis, the designs and applications of life on complex material system must be badly influenced. In fact, many applied effects of studies on complex system life have obviously proved this point up to now. In other words, modern computing techniques and implements are imprudently or blindly applied to complex system life before that the data are suitably and adequately disposed, so that it might be result in jumbled analysis. To solve these troublesome issues, the two advises are given as below: (1) predigesting data structure of complex material system life; (2) partitioning complex material system on life.

In analysis of complex system life, there is a kind of issue which that is not seriously investigated, and that is analysis of complex material system life under variable structures. Indeed, the disparate system behaviors are accordingly determined by the system structures, and foremost, the disparate system lives are also accordingly determined by the variable structures ${ }^{[5-8]}$. In this paper, the more rudimental issue will be discussed, that is, the analysis of system life are discriminated in two kinds of variable structures on system life indexes. At first, for the sake of the simple and convenient, the discriminant analysis of variable structure system on four subsystems is not straightly put in practice, and through the principal component analysis for the four life indexes of original subsystem, the two principal component indexes are picked up among the four indexes. The second, according to two kinds of variable structure based on the two indexes, the discriminant categorizing of the data 20 
groups are approximately categorized into two kinds. Thus, the discriminant categorizing on the empirical data and simulating data on variable structure life of complex material system is realized, and the subsequent analysis on system life might be expediently disposed. In fact, multivariable structures might also be easily implemented as the analogous tactics.

\section{Principal Component Analysis based on Empirical Data}

Data Pretreatments before Principal Component Analysis. Here, the topical circumstances of this paper are at first stated as below, the entire system consists of four subsystems, and shows itself as two kinds of variable structure with runtime and environment, and so on, that the running structure of the real system is not factitiously decided. For the four subsystems, 20 groups of their life indexes are gained by the empirical data and the simulating data and the evaluating and analyses, and the 20 groups of data have been standardized and shown in Table 1 for sake of the length saving of the paper.

In the Table 1, the most left row denotes the serial number of the data order by the empirical data and the simulating data and the evaluating and analyses, the most right row denotes the running categorization states of the system in $A$ and $B$ for the two kinds of variable structures. $X_{1}, X_{2}, X_{3}$, $X_{4}$ denote the four subsystems, and the data in the middle of Table 1 denote standardization data.

Table 1 Standardization data and categorization table on life indexes

\begin{tabular}{cccccc}
\hline $\begin{array}{c}\text { Serial } \\
\text { number }\end{array}$ & $X_{1}$ & $X_{2}$ & $X_{3}$ & $X_{4}$ & Categorization \\
\hline 1 & 334.1113 & 36.49658 & 11.6619 & 80.53768 & $A$ \\
2 & 716.7205 & 75.82216 & 12.04159 & 48.74299 & $A$ \\
3 & 774.505 & 58.13777 & 16.97056 & 40.81508 & $A$ \\
4 & 1295.376 & 59.5063 & 27.73085 & 155.1576 & $A$ \\
5 & 372.5112 & 39.40812 & 12.04159 & 125.7067 & $A$ \\
6 & 419.3877 & 34.1321 & 15.65248 & 121.8274 & $A$ \\
7 & 266.115 & 31.89044 & 10.63015 & 131.1859 & $A$ \\
8 & 539.6714 & 62.24147 & 11.04536 & 66.31791 & $A$ \\
9 & 302.0649 & 30.23243 & 12.72792 & 124.2157 & $A D$ \\
10 & 2244.484 & 73.40981 & 38.94868 & 114.121 & $A$ \\
11 & 845.9387 & 77.36924 & 13.92839 & 153.1038 & $B$ \\
12 & 396.0883 & 34.98571 & 14.42221 & 59.03624 & $B$ \\
13 & 389.6796 & 57.70615 & 8.602325 & 81.02737 & $B D$ \\
14 & 742.3631 & 70.29225 & 13.45362 & 129.8056 & $B$ \\
15 & 1060.331 & 85.42833 & 15.81139 & 115.6652 & $B$ \\
16 & 240.6368 & 21.84033 & 14.03567 & 164.0546 & $B D$ \\
17 & 303.7859 & 32.24903 & 12 & 172.875 & $B$ \\
18 & 383.3662 & 51.47815 & 9.486833 & 24.0755 & $B$ \\
19 & 249.73 & 39.45884 & 8.062258 & 81.25384 & $C B$ \\
20 & 899.154 & 71.44928 & 16.03122 & 83.57125 & $B$ \\
\hline & & & & &
\end{tabular}

Principal Component Analysis on Complex Material System Life. Let $X$ denote the data in the middle of Table 1 , then $X$ is a $20 \times 4$ matrix. Now, principal component analysis on the standardized 
index data of four subsystem life are given as the below where that are algorithmic steps and calculating achievement of the data.

(1)The original data are standardized as shown in Table 1, and denoted the matrix $X$;

(2) Calculating the relation matrix $R=X^{T} X$, and $R$ is a $4 \times 4$ matrix;

(3) Calculating the eigenvalues and eigenvectors of $R$;

(4)Based on the above results and the principle of principal component analysis, the summing contribution ratio of the front two principal components on their eigenvalues in the four principal components has reached beyond $89.85 \%$. Thus, the reasons are highly sufficient that the front two principal components consist basically of the full information on system life indexes, and the formulas of the front two principal components are shown as below:

$$
\begin{aligned}
& F_{1}=-0.9959 X_{1}+0.0596 X_{2}+0.0568 X_{3}+0.0362 X_{4} . \\
& F_{2}=0.6077 X_{1}+0.5660 X_{2}+0.0886 X_{3}+0.0142 X_{4}
\end{aligned}
$$

Where $F_{1}$ is the first principal component, and $F_{2}$ is the second principal component. On second thoughts, there are still highly sufficient reasons that the front two standardized indexes consist basically of the full information on the primary four life indexes according to the coefficients in the above two formulas. Therefore, the below categorizing analysis only is considered in the form of the front two standardized indexes on system life. Here, the routine test on statistics analysis is omitted, for the sake of the saving length of this paper.

\section{The Results on the Classification Discriminant of System Life}

The classification results about the two principal components. References are cited in the text just by square brackets $^{[1]}$. (If square brackets are not available, slashes may be used instead, e.g. /2/.) Two or more references at a time may be put in one set of brackets ${ }^{[3,4]}$. The references are to be numbered in the order in which they are cited in the text and are to be listed at the end of the contribution under a heading References, see our example below.

Now there are two kinds of states in system running, $G_{1}, G_{2}$ denote the two states, and the classification discriminant are put in practice with the above analysis, according to the front two standardized indexes on system reliability in Table 1. Here, the Euclidian distance is used in data categorization, that is, the Euclidian distance $D(Y, G)$, and the testing data denote $Y_{1}, Y_{2},{ }_{2} \mathrm{~K} Y_{20}$, and general $Y$. Through the averaging method from the prep-data, $G_{1}, G_{2}$ are quantitatively translated into $G_{1}=(79.849,36.726)^{T}$ and $G_{2}=(58.913,31.704)^{T}{ }^{[9-12]}$. Moreover, the discrimination criterion are noted as below:

(1) $Y \in G_{1}$, if $D\left(X, G_{1}\right)<D\left(X, G_{2}\right)$;

(2) $Y \in G_{2}$, if $D\left(X, G_{1}\right)>D\left(X, G_{2}\right)$;

(3) the undetermined, when $D\left(Y, G_{1}\right)=D\left(Y, G_{2}\right)$;

(4) $D(Y, G)=(Y-G)^{T} \Sigma^{-1}(Y-G)$, here $\Sigma^{-1}$ might be denoted as the relation matrix of the front two principal component vectors that the matrix is $2 \times 2$ non-singular one. The results of the classification discriminant on the data are shown in Table 1 , where $A D, B D$ denote the misjudged with the original belongs respectively to $A$ or $B$, moreover, $C B$ denotes the undetermined. Thus, according to Table 1 , the precise radio is $80 \%$, and the misjudged radio and the undetermined ratio are summed as $20 \%$. In fact, the ratio $20 \%$ is still reduced as the elaborating method is employed.

\section{Conclusions}

The conclusion of this paper was given as the following: (1) According to the principal component analysis and classification discriminant analysis, the data might be categorized as the two kinds of 
variable structure with runtime and environment based on system life indexes, the precise radio is $80 \%$, and the misjudged radio and the undetermined ratio are summed as $20 \%$; (2) The principal component analysis and classification discriminant analysis can be the same with the life analysis on variable structure and complex material system.

\section{Acknowledgements}

This work was financially supported by the National Natural Science Foundation of China (51001117), Pre-research Item (51327020102), NSF of Beijing (3132024, 3120001) and Distinguished Young Scholars of NSFC (51125023).

\section{References}

[1] Ma Kemao, Wang Zicai, Zhang Decheng: Electric Machines and Control, Vol. 5(3), (2001), p. 181.

[2] Hu Jianbo, Zhuang Kaiyu, in: Theory and Applications of Advanced Variable Structure Systems, edtied by Northwest Industrial University Press, XiAn (2008) .

[3] Hu Yaoming, in: Theory and Applications of Advanced Variable Structure Systems. edtied by Science Press, Beijing (2003).

[4] Jin Xing, Hong Yanji, in: Assessment Methods of System Reliability, edtied by National Defence Industry Press, Beijing (2005).

[5] Song Baowei, Wang Xiaojuan, in: Design and Analysis of System Reliability, edtied by Northwest Industrial University, Xian (2003).

[6] Xiao Gang, in: Monte Carlo Methods in System Reliability Analysis, edtied by Science Press, Beijing (2003).

[7] Yang Baochen, Zhang Shiying. Study on cointegration with structural changes [J]. Journal of Systems Engineering, 2002, 17(1):26 31.

[8] Sun Qinghua, Zhang Shiying. Structure change analysis of complex system [J]. Journal of Systems Engineering, 2000, 15(4):344 351.

[9] Yu Dan, Li Xuejing, Jiang Ningning: Journal of Systems Science and Mathematical Sciences, Vol. 27(2), (2007), p. 68.

[10] Yang Yuhang, Li Zhizhong, Zheng Li: Acta Simulata Systematica Sinica, Vol. 17(3), (2005), p. 710.

[11] Gao Ping, Wu Su,Jia Xisheng: Journal of System Simulation, Vol. 21(13), (2009), p. 4140.

[12] Gao Huixuan, in: Applied Multivariate Statistical Analysis, edtied by Beijing University Press, Beijing (2005). 